\title{
Time-driven interventions for affective disorders: on resonance and the Oberth effect
}

\author{
Felipe C. Argolo, ${ }^{1,2}{ }^{(1 D}$ Ary Gadelha,${ }^{1}$ Pedro M. Pan, ${ }^{1}$ Rodrigo A. Bressan ${ }^{1,2}$
}

Mood can be examined through the evolution of affective states in time, ${ }^{1}$ and unraveling these dynamics may provide insights for innovative interventions. Recent efforts describe domains of biological rhythms in mental disorders, including sleep, daily activities, social interactions and eating patterns. ${ }^{2}$

We illustrate how time-driven interventions can be used for controlled interference in clinical settings. Whereas the concept of benefiting from dynamical properties of systems is new to psychiatry, it is a common asset to medicine.

On the face of rhythms unsusceptible to cardioversion, specialists induce favorable patterns using drugs (e.g., amiodarone). Also, the electrical shock is synchronized with a sweet-spot in the electrocardiogram's R wave, preventing ventricular fibrillation.3,4 Gynecologists monitor menstrual cycle in order to deliver precise care, from basic birth-planning to varying-dose contraceptive drugs. ${ }^{5}$

Previous works showed similar dynamical traits in behavior: for example, predicted and obtained rewards relate to the momentum of happiness measures. ${ }^{1}$ Can this perspective better inform clinicians to design interventions?

We often look for optimal schedules, whether in focused (e.g., infused ketamine or electroconvulsive therapy) or long-duration (e.g. combining standard pharmacotherapy with psychotherapy) treatment. Increasing familiarity with psychopathology dynamics is expected to fine-tune treatment. When searching for optimal moments to intervene, valuable insights may come from examining analogous phenomena. Specifically, one may synchronize interventions with natural trajectories of affect, using regularly visited states as a compass to improve efficiency. ${ }^{6}$

In orbital mechanics, the Oberth effect poses that an aircraft gains more kinetic energy when accelerating at the nearest point to a central body, where potential is lowest and speed is highest. European Swedishdesigned satellite SMART-1 (2003) made use of these properties to spiral out of Earth's orbit until it reached the moon (2006), as did India's recent lunar mission Chandrayanan-2 (2019).

A more intuitive example can be observed in a child's toy: the playground swing. In order to optimize efforts, adults push the seat synchronizing it with the natural motion of the pendulum when going down-and-forward. This maneuver uses gravitational forces to maximize kinetic energy and make transitions to orbits of larger amplitudes.

We can make use of a similar timewise rationale to fine-tune treatment. For instance, the synergistic effect of cognitive behavioral therapy (CBT) and antidepressants may be optimized by starting CBT when drug-induced susceptibility of mood to perturbations is at maximum. Previous results suggest that it is possible to modulate biological factors (e.g., cortisol) level through behavioral changes in subjects with depression. ${ }^{7}$

Psychiatry is moving fast. We believe that examining the dynamics of affective states in time can give us insightful new ways to help people with mental disorders.

\footnotetext{
${ }^{1}$ Departamento de Psiquiatria, Universidade Federal de São Paulo (UNIFESP), São Paulo, SP, Brazil. ${ }^{2}$ King's College London, London, UK. Submitted Aug 08 2019, accepted for publication Oct 062019.

Suggested citation: Argolo FC, Gadelha A, Pan PM, Bressan RA. Time-driven interventions for affective disorders: on resonance and the Oberth effect. Trends Psychiatry Psychother. 2020;42(2):113-114. http://dx.doi.org/10.1590/2237-6089-2019-0067
} 


\section{Acknowledgements}

Felipe C. Argolo is sponsored by a PhD scholarship from Conselho Nacional de Desenvolvimento Científico e Tecnológico (CNPq)/Coordenação de Aperfeiçoamento de Pessoal de Nível Superior (CAPES).

\section{Disclosure}

No conflicts of interest declared concerning the publication of this article.

\section{References}

1. Eldar E, Rutledge RB, Dolan RJ, Niv Y. Mood as representation of momentum. Trends Cogn Sci. 2016;20:15-24. https://doi. org/10.1016/j.tics.2015.07.010
2. Berny T, Jansen K, Cardoso TA, Mondin TC, Silva RA, Souza LD, et al. Construction of a biological rhythm assessment scale for children. Trends Psychiatry Psychother. 2018;40:53-60.

3. Zipes DP, Jackman WM, Heger JJ, Chilson DA, Browne KF, Naccarelli GV, et. al. Clinical transvenous cardioversion of recurrent life-threatening ventricular tachyarrhythmias: Low energy synchronized cardioversion of ventricular tachycardia and termination of ventricular fibrillation in patients using a catheter electrode. Am Heart J. 1982;103:789-94.

4. Garfinkel A, Spano ML, Ditto WL, Weiss JN. Controlling cardiac chaos. Science. 1992;257:1230-5.

5. Alam MA, Ali R, Al-Jenoobi FI, Al-mohizea AM. Advanced oral contraceptive regimens and their management. Am J Pharm. 2014;6:e103-14.

6. Friston KJ, Redish AD, Gordon JA. Computational nosology and precision psychiatry. Comput Psychiatry. 2017;1:2-23

7. Beserra AHN, Kameda P, Deslandes AC, Schuch FB, Laks J, Moraes HS. Can physical exercise modulate cortisol level in subjects with depression? A systematic review and meta-analysis. Trends Psychiatry Psychother. 2018;40:360-8.

\section{Correspondence:}

Felipe Coelho Argolo

Pedro de Toledo, 669, 3rd floor

04039-032 - São Paulo, SP - Brazil

E-mail: felipe.c.argolo@protonmail.com 